\title{
Optimal control of electrical and thermal energy storage to minimise time-of-use electricity costs
}

\author{
L. R. Cirocco, ${ }^{\mathrm{a}}$ J. Boland, ${ }^{\mathrm{a}}$ M. Belusko, ${ }^{\mathrm{b}}$ F. Bruno ${ }^{\mathrm{b}}$ and P. Pudney ${ }^{\mathrm{a}}$ \\ ${ }^{a}$ Centre for Industrial and Applied Mathematics, \\ School of Information Technology and Mathematical Sciences / Barbara Hardy Institute, \\ University of South Australia, Mawson Lakes Boulevard, Mawson Lakes, SA, 5095, Australia \\ ${ }^{\mathrm{b}}$ School of Engineering / Barbara Hardy Institute, University of South Australia, Australia \\ Email: luigi.cirocco@mymail.unisa.edu.au
}

\begin{abstract}
The advent of new electricity metering technologies means that consumers can now be billed for electricity using prices that vary with time-of-use. At the same time, new electrical energy storage systems and thermal energy storage systems give consumers an opportunity to control when they import electricity from the grid.

In this paper we construct a power flow model of a system with both electrical and thermal energy storage, and use Pontryagin's principle to derive necessary conditions for a control strategy that minimises the cost of energy from the grid. The optimal control has just three control modes for each storage system: charge, off, and discharge. Which mode should be used at any instant for each of the storage system depends on the price of electricity relative to two critical prices for each of the storage systems. We use a realistic example to illustrate how the critical prices for each subsystem can be determined, and to determine the ideal capacity of each storage system.
\end{abstract}

Keywords: Electrical energy storage, thermal energy storage, optimal control, time-of-use tariff 
L. R. Cirocco et al., Optimal control of electrical and thermal energy storage ...

\section{INTRODUCTION}

Traditionally, electricity consumers pay a fixed rate for the electricity they consume, irrespective of when they consume it. This is despite the fact that the cost of generating and distributing electricity varies considerably with demand. With the advent of new electricity meters that measure when electricity is consumed as well as how much electricity is consumed, new tariffs are being introduced that allow customers to be charged higher rates during peak periods when demand is usually high, and lower rates during off-peak periods.

At the same time, generous feed-in tariffs have encouraged many consumers to install rooftop photovoltaic systems that allow them generate electricity and be paid for excess generation fed back into the grid. As feed-in tariffs reduce and the cost of energy storage systems drops, it will become more cost-effective to store any excess energy generated rather than export it for a low price only to import energy later at a significantly higher cost.

Previously we have considered the optimal control of a large concentrating solar thermal plant with storage, to maximise the income from exporting energy into the wholesale energy market with time-varying prices (Cirocco et al. 2015). In this paper we consider how a consumer can use both electrical energy storage systems and thermal energy storage systems to minimise the cost of energy from the grid when the price of electricity from the grid varies with time.

A review article by Sabihuddin et al. (2014) compares electrical and thermal storage technologies that can be used for regulating power quality, providing bridging power, and for energy management or load smoothing.

Optimal control of thermal systems is widely documented. Henze et al. (2011) uses mathematical programming to minimise energy and demand costs for an ice storage system used to cool a commercial building. Although energy use increases due to losses in the storage system, there is a significant reduction in the demand related costs. Bakos (2000) uses Pontryagin's principle to minimise the cost of electrical energy for underfloor space heating, with a passive solar thermal Trombe wall to provide for heat capture during the day. LeBreux et al. (2009) describes a fuzzy logic feed forward controller with weather forecasting for controlling for space heating with a passively heated thermal mass and separate thermal storage using electrically heated ceramic bricks. Candanedo et al. (2013) compares a model-based predictive control algorithm against benchmark storage priority and chiller priority heuristics for space cooling using thermal storage, demonstrating an improvement in cost savings ranging from 5\%-30\% from the benchmark controls.

In this paper we consider a consumer who has electrical loads, an electrical energy storage system, thermal loads where the thermal energy is generated from electrical energy, and a thermal storage system. We formulate and solve the problem of controlling the electrical and thermal storage systems to minimise the cost of electricity when the price of electricity varies with time of use.

\section{SYSTEM MODEL AND PROBLEM FORMULATION}

Figure 1 depicts the possible flows of electrical and thermal power for a grid-connected consumer with both electrical and thermal energy storage systems and renewable energy sources available "behind the meter" where the consumer is metered for net energy import or export. The power flows all vary with time and are all non-negative. They are as follows:

- $G_{\mathrm{imp}}$ is electrical power imported from the grid, and is determined from other power flows in the system

- $G_{\text {exp }}$ is electrical power that flows back to the grid, and is determined from other power flows in the system

- $R_{\mathrm{e}}$ is electrical power supplied from local renewable energy sources such as photovoltaic panels, and is a given function of time

- $L_{\mathrm{e}}$ is the electrical load, and is a given function of time

- $P_{\text {et }}$ is the electrical power used to generate heating or cooling, and depends on downstream thermal power flows

- $L_{\mathrm{t}}$ is the thermal load, and is a given function of time

- $C_{\mathrm{e}}$ is electrical power used to charge the electrical storage system, and is a time-varying control 
L. R. Cirocco et al., Optimal control of electrical and thermal energy storage ...

- $D_{\mathrm{e}}$ is electrical power discharged from the electrical storage system, and is a time-varying control

- $C_{\mathrm{t}}$ is thermal power used to charge the thermal storage system, and is a time-varying control

- $D_{\mathrm{t}}$ is thermal power discharged from the electrical storage system, and is a time-varying control.

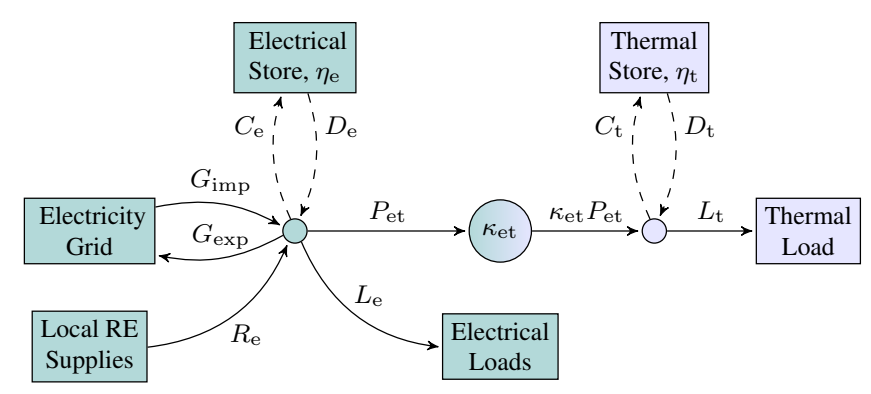

Figure 1. Power flows for a consumer with renewable and grid connected electricity supplies fitted with both electrical and thermal storage systems for servicing a mix of electrical and thermal loads

Electrical power is converted to thermal power by a heat pump or compressor, which has a constant coefficient of performance $\kappa_{\text {et }} \geq 1$ for the purposes of this initial investigation we avoid the added complexity of varying this parameter with respect to ambient temperature in order to establish the salient aspects of an optimal control strategy.

At the first electrical distribution node, the power flows are related by

$$
R_{\mathrm{e}}+G_{\mathrm{imp}}-G_{\mathrm{exp}}-L_{\mathrm{e}}-C_{\mathrm{e}}+D_{\mathrm{e}}-P_{\mathrm{et}}=0 .
$$

The thermal subsystem input electrical power, $P_{\text {et }}$, is dependent on the two thermal storage controls and the given thermal load, and is given by

$$
P_{\mathrm{et}}=\left(L_{\mathrm{t}}+C_{\mathrm{t}}-D_{\mathrm{t}}\right) / \kappa_{\mathrm{et}} .
$$

We wish to minimise the cost of energy for this system during some time interval $[0, T]$. The cost $\pi_{\mathrm{i}}$ of imported electrical energy and the price $\pi_{\mathrm{e}}$ paid for exported electrical energy are both given functions of time, and so the total cost of operating the system is

$$
J\left(G_{\mathrm{imp}}, G_{\mathrm{exp}}, \pi_{\mathrm{i}}, \pi_{\mathrm{e}}, t\right)=\int_{0}^{T}\left(\pi_{\mathrm{i}} G_{\mathrm{imp}}-\pi_{\mathrm{e}} G_{\mathrm{exp}}\right) d t .
$$

If we use (1) and (2) to write $G_{\mathrm{imp}}$ and $P_{\mathrm{et}}$ in terms of the remaining power flows, the objective function (3) can be expressed in terms of the given power flows and the introduced control flows as

$$
\begin{aligned}
J & \left(\pi_{\mathrm{i}}, \pi_{\mathrm{e}}, R_{\mathrm{e}}, L_{\mathrm{e}}, L_{\mathrm{t}}, C_{\mathrm{e}}, D_{\mathrm{e}}, C_{\mathrm{t}}, D_{\mathrm{t}}, G_{\mathrm{exp}}, t\right) \\
& =\int_{0}^{T}\left(\pi_{\mathrm{i}}\left(G_{\exp }-R_{\mathrm{e}}+\left(L_{\mathrm{e}}+C_{\mathrm{e}}-D_{\mathrm{e}}\right)+\left(\frac{L_{\mathrm{t}}+C_{\mathrm{t}}-D_{\mathrm{t}}}{\kappa_{\mathrm{et}}}\right)\right)-\pi_{\mathrm{e}} G_{\text {exp }}\right) d t \rightarrow \min .
\end{aligned}
$$

The energy levels in the electrical and thermal stores are given by the differential equations

$$
\frac{d}{d t} Q_{\mathrm{e}}=\eta_{\mathrm{e}} C_{\mathrm{e}}-D_{\mathrm{e}}, \quad Q_{\mathrm{e}}(0)=Q_{\mathrm{e} 0}
$$

and

$$
\frac{d}{d t} Q_{\mathrm{t}}=\eta_{\mathrm{t}} C_{\mathrm{t}}-D_{\mathrm{t}}, \quad Q_{\mathrm{t}}(0)=Q_{\mathrm{t} 0}
$$

where $\eta_{e}$ and $\eta_{t}$ are the constant efficiencies of the electrical and thermal storage systems respectively. In practice the stored energy in each system would be constrained by lower and upper bounds. We will assume 
that storage capacity constraints are never active, so that we can determine the ideal capacity of the electrical and thermal storage systems. However, we will impose constraints

$$
\begin{aligned}
& Q_{\mathrm{e} 0} \leq Q_{\mathrm{e}}(T) \\
& Q_{\mathrm{t} 0} \leq Q_{\mathrm{t}}(T)
\end{aligned}
$$

which ensure that the energy stored in each storage system at time $t=T$ is at least as much as stored at time $t=0$, so that the system can run indefinitely.

We impose the following limits on the power flows:

$$
\begin{aligned}
& 0 \leq G_{\mathrm{imp}} \leq \bar{G}_{\mathrm{imp}} \\
& 0 \leq G_{\mathrm{exp}} \leq \bar{G}_{\mathrm{exp}} \\
& 0 \leq R_{\mathrm{e}} \leq \bar{R}_{\mathrm{e}} \\
& 0 \leq C_{\mathrm{e}} \leq \bar{C}_{\mathrm{e}} \\
& 0 \leq D_{\mathrm{e}} \leq \bar{D}_{\mathrm{e}} \\
& 0 \leq C_{\mathrm{t}} \leq \bar{C}_{\mathrm{t}} \\
& 0 \leq D_{\mathrm{t}} \leq \bar{D}_{\mathrm{t}} \\
& 0 \leq L_{\mathrm{t}}+C_{\mathrm{t}}-D_{\mathrm{t}} \leq \kappa_{\mathrm{et}} \bar{P}_{\mathrm{et}} .
\end{aligned}
$$

\section{NECESSARY CONDITIONS FOR OPTIMALITY}

We use Pontryagin's principle to find necessary conditions for an optimal control. We first form a Hamiltonian, to be maximised:

$$
H\left(R_{\mathrm{e}}, \pi_{\mathrm{i}}, \pi_{\mathrm{e}}, L_{\mathrm{e}}, L_{\mathrm{t}}, C_{\mathrm{e}}, D_{\mathrm{e}}, G_{\mathrm{exp}}, C_{\mathrm{t}}, D_{\mathrm{t}}, Q_{\mathrm{e}}, Q_{\mathrm{t}}, \lambda_{\mathrm{e}}, \lambda_{\mathrm{t}}, t\right)=-J+\lambda_{\mathrm{e}} \frac{d}{d t} Q_{\mathrm{e}}+\lambda_{\mathrm{t}} \frac{d}{d t} Q_{\mathrm{t}}
$$

or

$$
\begin{aligned}
H[t]= & \pi_{\mathrm{i}} R_{\mathrm{e}}-\pi_{\mathrm{i}} L_{\mathrm{e}}-\left(\pi_{\mathrm{i}} / \kappa_{\mathrm{et}}\right) L_{\mathrm{t}}+\left(\pi_{\mathrm{e}}-\pi_{\mathrm{i}}\right) G_{\exp }+\left(\eta_{\mathrm{e}} \lambda_{\mathrm{e}}-\pi_{\mathrm{i}}\right) C_{\mathrm{e}}+\left(\pi_{\mathrm{i}}-\lambda_{\mathrm{e}}\right) D_{\mathrm{e}} \\
& +\left(\eta_{\mathrm{t}} \lambda_{\mathrm{t}}-\left(\pi_{\mathrm{i}} / \kappa_{\mathrm{et}}\right)\right) C_{\mathrm{t}}+\left(\left(\pi_{\mathrm{i}} / \kappa_{\mathrm{et}}\right)-\lambda_{\mathrm{t}}\right) D_{\mathrm{t}}
\end{aligned}
$$

The controls of our system are the exported power $G_{\mathrm{exp}}$, the electrical storage flows $C_{\mathrm{e}}$ and $D_{\mathrm{e}}$, and the thermal storage flows $C_{\mathrm{t}}$ and $D_{\mathrm{t}}$. To be optimal, these controls must be chosen to maximise the Hamiltonian. A preliminary observation is that if $\pi_{\mathrm{e}}<\pi_{\mathrm{i}}$, as is almost always the case, the Hamiltonian is maximised when $G_{\text {exp }}$ is minimised.

To further simplify our analysis, we will consider a system with no renewable power input and where the export price is set to zero so that there are no opportunities for arbitrage. By limiting the investigation to this simpler form of problem, the associated Hamiltonian becomes

$$
\begin{aligned}
H[t]= & -\pi_{\mathrm{i}} L_{\mathrm{e}}-\left(\pi_{\mathrm{i}} / \kappa_{\mathrm{et}}\right) L_{\mathrm{t}}+\left(\eta_{\mathrm{e}} \lambda_{\mathrm{e}}-\pi_{\mathrm{i}}\right) C_{\mathrm{e}}+\left(\pi_{\mathrm{i}}-\lambda_{\mathrm{e}}\right) D_{\mathrm{e}} \\
& +\left(\eta_{\mathrm{t}} \lambda_{\mathrm{t}}-\left(\pi_{\mathrm{i}} / \kappa_{\mathrm{et}}\right)\right) C_{\mathrm{t}}+\left(\left(\pi_{\mathrm{i}} / \kappa_{\mathrm{et}}\right)-\lambda_{\mathrm{t}}\right) D_{\mathrm{t}} .
\end{aligned}
$$

The evolution of the adjoint variables $\lambda_{\mathrm{e}}$ and $\lambda_{\mathrm{t}}$ is given by

$$
\frac{d \lambda_{\mathrm{e}}}{d t}=-\frac{\partial H[t]}{\partial Q_{\mathrm{e}}}=0 \Longrightarrow \lambda_{\mathrm{e}}^{*} \text { is constant }
$$

and

$$
\frac{d \lambda_{\mathrm{t}}}{d t}=-\frac{\partial H[t]}{\partial Q_{\mathrm{t}}}=0 \Longrightarrow \lambda_{\mathrm{t}}^{*} \text { is constant. }
$$

The optimal adjoint values $\lambda_{\mathrm{e}}^{*}$ and $\lambda_{\mathrm{t}}^{*}$ are constant for both forms of the Hamiltonian, (17) and (18).

For the simplified problem with Hamiltonian (18), the optimal controls for the electrical energy storage system depend on the value of the price $\pi_{\mathrm{i}}$ relative to the optimal adjoint value $\lambda_{\mathrm{e}}^{*}$, as shown in Table 1 . 
L. R. Cirocco et al., Optimal control of electrical and thermal energy storage ...

Table 1. Optimal control modes for the electrical storage system

\begin{tabular}{lccc} 
mode & condition & $C_{\mathrm{e}}$ & $D_{\mathrm{e}}$ \\
\hline Charge & $\pi_{\mathrm{i}}<\eta_{\mathrm{e}} \lambda_{\mathrm{e}}^{*}$ & $\max$ & $\min$ \\
Off & $\eta_{\mathrm{e}} \lambda_{\mathrm{e}}^{*}<\pi_{\mathrm{i}}<\lambda_{\mathrm{e}}^{*}$ & $\min$ & $\min$ \\
Discharge & $\lambda_{\mathrm{e}}^{*}<\pi_{\mathrm{i}}$ & $\min$ & $\max$
\end{tabular}

Table 2. Optimal control modes for the thermal storage system

\begin{tabular}{lccc} 
mode & condition & $C_{\mathrm{t}}$ & $D_{\mathrm{t}}$ \\
\hline Charge & $\pi_{\mathrm{i}}<\eta_{\mathrm{t}} \kappa_{\mathrm{et}} \lambda_{\mathrm{t}}^{*}$ & $\max$ & $\min$ \\
Off & $\eta_{\mathrm{t}} \kappa_{\mathrm{et}} \lambda_{\mathrm{t}}^{*}<\pi_{\mathrm{i}}<\kappa_{\mathrm{et}} \lambda_{\mathrm{t}}^{*}$ & $\min$ & $\min$ \\
Discharge & $\kappa_{\mathrm{et}} \lambda_{\mathrm{t}}^{*}<\pi_{\mathrm{i}}$ & $\min$ & $\max$
\end{tabular}

Similarly, the optimal controls for the thermal energy storage system depend on the value of the price $\pi_{\mathrm{i}}$ relative to $\kappa_{\text {et }} \lambda_{\mathrm{t}}^{*}$, as shown in Table 2 .

It appears that the optimal controls for the two storage systems are independent, but this is not quite the case. Consider a scenario where we need to discharge the electrical store, and we are not allowed to export power. If the electrical load is low then the amount we can discharge from the electrical store will depend on $P_{\text {et }}$, which will in turn depend on the thermal load and on whether we are charging or discharging the thermal store.

There are situations where further analysis is required to determine the optimal control. To illustrate this, consider the further simplified system with ideal storage efficiencies $\eta_{\mathrm{e}}=\eta_{\mathrm{t}}=1$. In this case each store must be either charging or discharging - there is no 'off' mode. There are six possible combinations of electrical and thermal storage controls, depending on whether $\kappa_{\mathrm{et}} \lambda_{\mathrm{t}}^{*}$ is bigger or smaller than $\lambda_{\mathrm{e}}^{*}$. These cases are illustrated in Figure 2 .

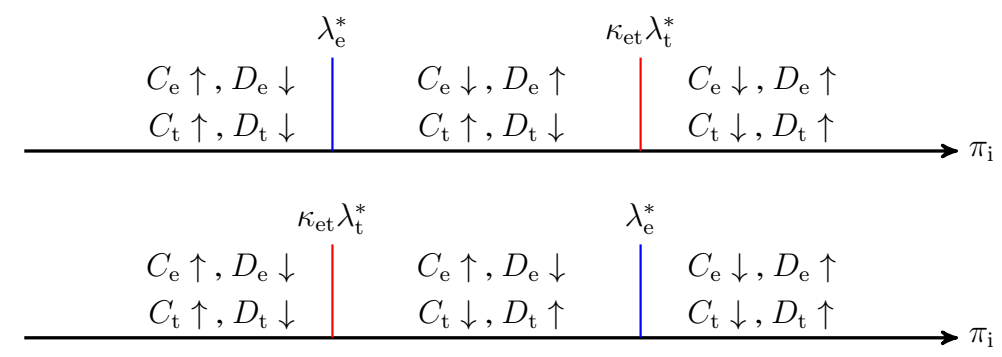

Figure 2. Combinations of optimal control modes for an import price $\pi_{\mathrm{i}}$ relative to the adjoint variables $\lambda_{\mathrm{e}}^{*}<\kappa_{\mathrm{et}} \lambda_{\mathrm{t}}^{*}$ (upper) and $\kappa_{\mathrm{et}} \lambda_{\mathrm{t}}^{*}<\lambda_{\mathrm{e}}^{*}$ (lower) for ideal storage efficiencies $\eta_{\mathrm{e}}=\eta_{\mathrm{t}}=1$ and electro-thermal power conversion factor $\kappa_{\mathrm{et}}$. The arrows indicate whether the control should be minimised or maximised.

Now consider the two cases, depicted on the right of Figure 2, where charging of each storage system is to be minimised and discharging of each storage system is to be maximised. If the total load is sufficiently small that it can be met without importing electricity then $G_{\mathrm{imp}}$ will be set to zero, and we must set $D_{\mathrm{e}}$ and $D_{\mathrm{t}}$ so that

$$
D_{\mathrm{e}}+D_{\mathrm{t}} / \kappa_{\mathrm{et}}=L_{\mathrm{e}}+L_{\mathrm{t}} / \kappa_{\mathrm{et}} .
$$

If the loads are sufficiently small that both loads can be met by discharging the electrical store only $\left(L_{\mathrm{e}}+\right.$ $\left.L_{\mathrm{t}} / \kappa_{\mathrm{et}}<\bar{D}_{\mathrm{e}}\right)$ and the thermal load can be met by discharging the thermal store only $\left(L_{\mathrm{t}}<\bar{D}_{\mathrm{t}}\right)$ then two possible control strategies are:

- use the electrical store to meet the electrical load $\left(D_{\mathrm{e}}=L_{\mathrm{e}}\right)$ and the thermal store to meet the thermal load $\left(D_{\mathrm{t}}=L_{\mathrm{t}}\right)$, in which case the Hamiltonian is $H_{\mathrm{a}}=-\lambda_{\mathrm{e}} L_{\mathrm{e}}-\lambda_{\mathrm{t}} L_{\mathrm{t}}$ 
L. R. Cirocco et al., Optimal control of electrical and thermal energy storage ...
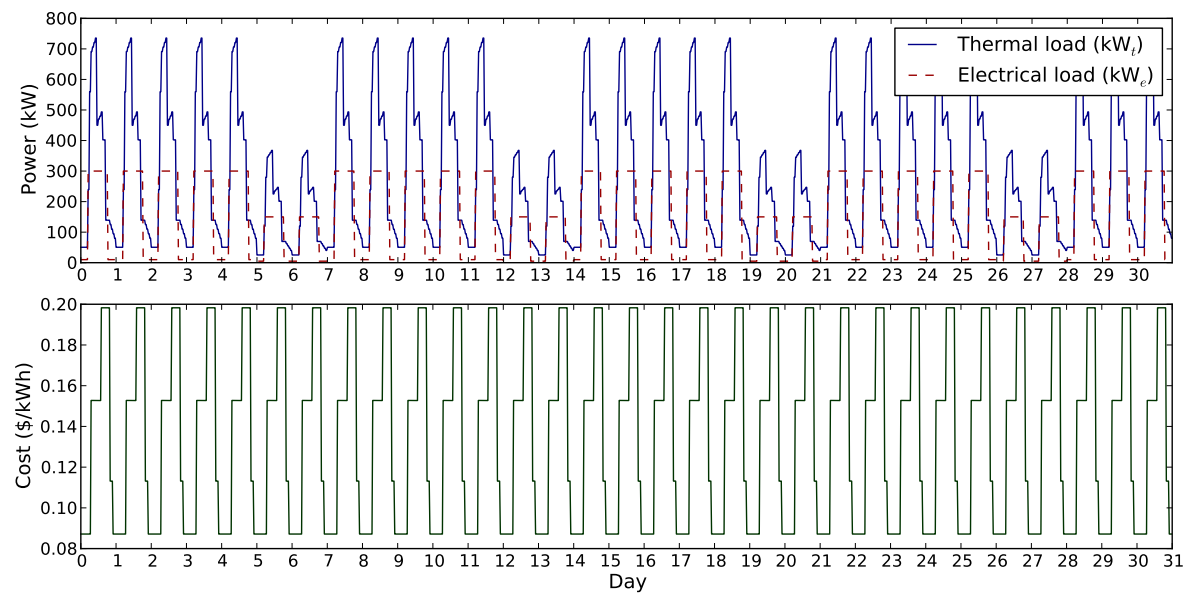

Figure 3. Load profiles (top) and price profile (bottom) for our example.

- use the electrical store to meet both the electrical and thermal loads $\left(D_{\mathrm{e}}=L_{\mathrm{e}}+L_{\mathrm{t}} / \kappa_{\mathrm{et}}, D_{\mathrm{t}}=0\right)$, in which case he Hamiltonian is $H_{\mathrm{b}}=-\lambda_{\mathrm{e}} L_{\mathrm{e}}-\lambda_{\mathrm{e}} / \kappa_{\mathrm{et}} L_{\mathrm{t}}$.

If $\lambda_{\mathrm{e}} / \kappa_{\mathrm{et}}<\lambda_{\mathrm{t}}$ then $H_{\mathrm{a}}<H_{\mathrm{b}}$ and the first option is better, otherwise the second option is better.

\section{Algorithm DESCRIPTION AND EXAMPLE}

We will illustrate the construction of a control sequence meeting the necessary conditions for an optimal control using an example where export to the grid is not allowed and where there is no renewable power. Figure 3 shows the electrical and thermal load profiles, and the price profile, for a dairy processing plant over a 31-day period. Electrical storage efficiency is $\eta_{\mathrm{e}}=0.8$, thermal storage efficiency is $\eta_{\mathrm{t}}=0.95$ and electro-thermal power conversion factor is $\kappa_{\mathrm{et}}=2.8$.

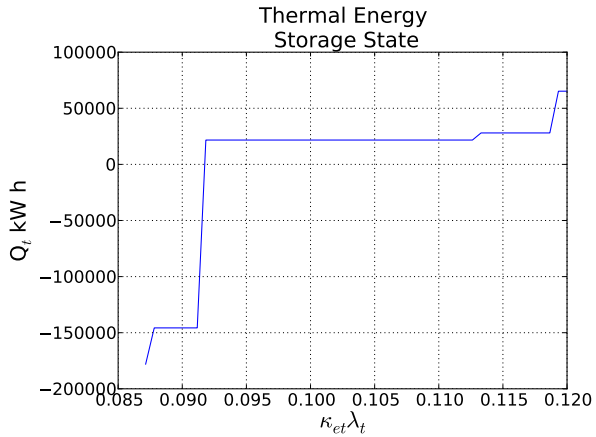

Figure 4. Final thermal store state as a function of $\kappa_{\mathrm{et}} \lambda_{\mathrm{t}}$.

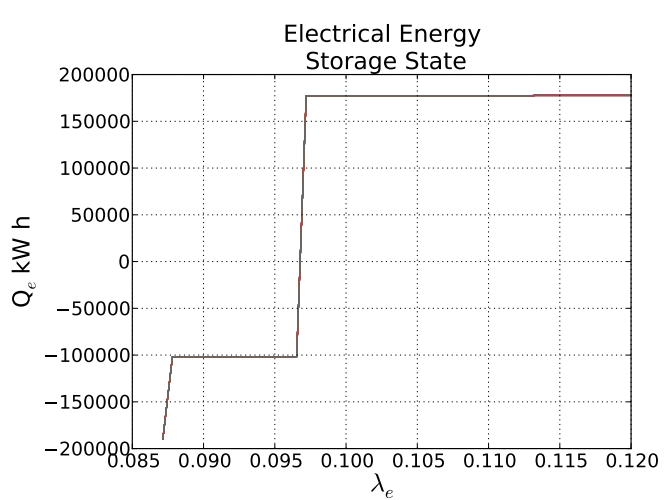

Figure 5. Electrical storage state as a function of adjoint variable $\lambda_{\mathrm{e}}$ for $\kappa_{\mathrm{et}} \lambda_{\mathrm{t}}^{*}=0.09173357$.

For any given pair $\left(\lambda_{\mathrm{e}}, \lambda_{\mathrm{t}}\right)$ we construct a control sequence by first using the control modes from Table 2 to set $C_{\mathrm{t}}$ and $D_{\mathrm{t}}$ to meet the thermal load, then calculate $P_{\mathrm{et}}$, then use the control modes from Table 1 to set $C_{\mathrm{e}}$ and $D_{\mathrm{e}}$ and hence calculate $G_{\mathrm{imp}}$. Each pair $\left(\lambda_{\mathrm{e}}, \lambda_{\mathrm{t}}\right)$ results in a final state $\left(Q_{\mathrm{e}}(T), Q_{\mathrm{t}}(T)\right)$. The lowest cost strategy will have $Q_{\mathrm{e}}(T)=Q_{\mathrm{e} 0}$ and $Q_{\mathrm{t}}(T)=Q_{\mathrm{t} 0}$.

Because we have chosen to meet the thermal loads first, the final state $Q_{\mathrm{t}}(T)$ of the thermal store will depend only on $\lambda_{\mathrm{t}}$, as shown in Figure 4. In this example we start with $Q_{\mathrm{t} 0}=0$, so wish to finish with $Q_{\mathrm{t}}(T)=0$; we need to set $\lambda_{\mathrm{t}}=0.09173 / \kappa_{\mathrm{et}}$.

With $\lambda_{\mathrm{t}}$ set, we now search for a value of $\lambda_{\mathrm{e}}$ that gives $Q_{\mathrm{e}}(T)=0$, as shown in Figure 5

Figure 6 shows the energy stored in the electrical and thermal stores for the resulting control profile, which 
L. R. Cirocco et al., Optimal control of electrical and thermal energy storage ...

also indicates the storage capacities required for the electrical and thermal stores.

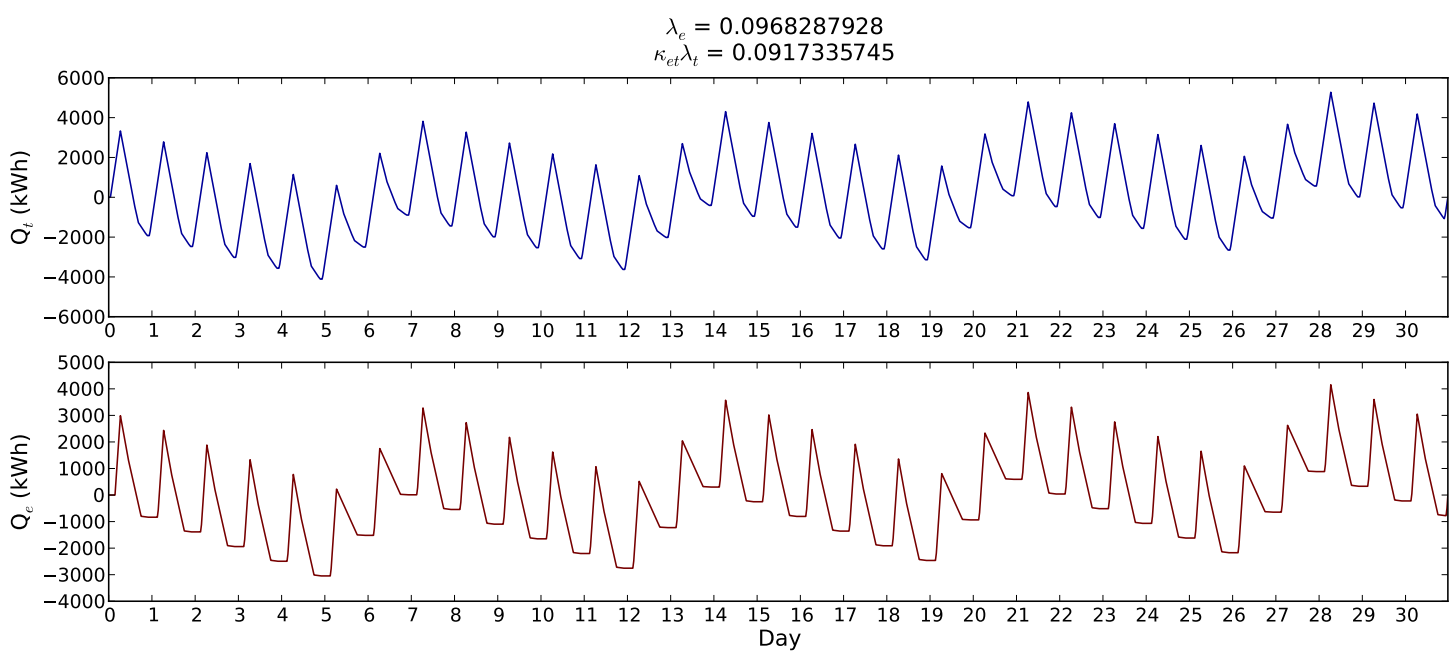

Figure 6. Stored energy profiles for our control profile.

\section{Conclusion}

We have formulated the problem of controlling an energy system with electrical and thermal energy storage when the cost of grid electricity varies with time of use, and used Pontryagin's principle to determine necessary conditions for an optimal control when grid export is not permitted and there is no local energy supply.

Each storage system has three possible control modes: charge, off, and discharge. The optimal mode for each storage system at any instant depends on the price of electricity relative to two critical prices-one for the electrical storage system and one for the thermal storage system.

We have used an example to illustrate how a control sequence satisfying the necessary conditions for an optimal control can be constructed. But we have also shown that further analysis is required to find the optimal control, and to prove uniqueness. Future work will also investigate the effect of storage capacity constraints on the optimal control.

\section{ACKNOWLEDGEMENT}

Luigi Cirocco would like to thank the School of Information Technology and Mathematical Sciences and the Barbara Hardy Institute, University of South Australia, for financial support.

\section{REFERENCES}

Bakos, G. (2000, April). Energy management method for auxiliary energy saving in a passive-solar-heated residence using low-cost off-peak electricity. Energy Build. 31(3), 237-241.

Candanedo, J., V. Dehkordi, and M. Stylianou (2013, November). Model-based predictive control of an ice storage device in a building cooling system. Appl. Energy 111, 1032-1045.

Cirocco, L. R., J. Boland, M. Belusko, F. Bruno, and P. Pudney (2015, January). Controlling stored energy in a concentrating solar thermal power plant to maximise revenue. IET Renew. Power Gener. 9(4), 379-388.

Henze, G. P., M. Krarti, and M. J. Brandemuehl (2011, February). A Simulation Environment for the Analysis of Ice Storage Controls. HVAC\&R Res.

LeBreux, M., M. Lacroix, and G. Lachiver (2009, March). Control of a hybrid solar/electric thermal energy storage system. Int. J. Therm. Sci. 48(3), 645-654.

Sabihuddin, S., A. Kiprakis, and M. Mueller (2014, December). A Numerical and Graphical Review of Energy Storage Technologies. Energies 8(1), 172-216. 